\title{
Correspondence between the surface integral and linear combination of atomic orbitals methods for ionic-covalent interactions in mutual neutralization processes involving $\mathrm{H}^{-} / \mathrm{D}^{-}$
}

\author{
Paul S. Barklem (1) \\ Theoretical Astrophysics, Department of Physics and Astronomy, Uppsala University, Box 516, SE-751 20 Uppsala, Sweden
}

(Received 16 September 2021; accepted 23 November 2021; published 13 December 2021)

\begin{abstract}
The surface integral method for estimating ionic-covalent interactions in diatomic systems been successful in producing cross sections for mutual neutralization $(\mathrm{MN})$ in reasonable agreement with experimental results for branching fractions between final states in systems such as $\mathrm{O}^{+} / \mathrm{O}^{-}$and $\mathrm{N}^{+} / \mathrm{O}^{-}$. However, for simpler cases of $\mathrm{MN}$ involving $\mathrm{H}^{-}$or $\mathrm{D}^{-}$, such as $\mathrm{Li}^{+} / \mathrm{D}^{-}$and $\mathrm{Na}^{+} / \mathrm{D}^{-}$, it has not produced results that are in agreement with experiments and other theoretical calculations; in particular, for $\mathrm{Li}^{+} / \mathrm{D}^{-}$calculations predict the wrong ordering of importance of final channels, including the incorrect most populated channel. The reason for this anomaly is investigated, and a leading constant to the asymptotic $\mathrm{H}^{-}$wave function is found that is different by roughly a factor of $1 / \sqrt{2}$ from that which has been used in previous calculations with the surface integral method involving $\mathrm{H}^{-}$or $\mathrm{D}^{-}$. With this correction, far better agreement with both experimental results and calculations with full quantum and linear combination of atomic orbitals (LCAO) methods is obtained. Further, it is shown that the surface integral method and LCAO methods have the same asymptotic behavior, in contrast to previous claims. This result suggests the surface integral method, which is comparatively easy to calculate, has greater potential for estimating $\mathrm{MN}$ processes than earlier comparisons had suggested.
\end{abstract}

DOI: 10.1103/PhysRevA.104.062806

\section{INTRODUCTION}

Charge transfer, in which an electron moves from one atom or ion to another during a collision, is a fundamental atomic process. Mutual neutralization (MN), with the corresponding reverse process ion-pair production

$$
\mathrm{A}^{+}+\mathrm{B}^{-} \rightleftharpoons \mathrm{A}^{*}+\mathrm{B},
$$

is an important special case, and methods for estimating cross sections are important for interpreting experimental results and testing our understanding of the physical mechanisms involved [1-3], as well as modeling various plasma environments such as planetary and planetary-satellite atmospheres [4], including earth's ionosphere [5] and stellar atmospheres [6,7], and being important for diagnostics of and fusion plasma applications and high-energy physics experiments employing negative-ion sources [8]. The transferred electron is captured into specific excited states $\mathrm{A}^{*}$, and thus affects the distribution of populations of states of atom $\mathrm{A}$ and its observed spectrum. These processes occur predominantly through interactions between ionic $\left(\mathrm{A}^{+}+\mathrm{B}^{-}\right)$and covalent $\left(\mathrm{A}^{*}+\mathrm{B}\right)$ configurations at avoided crossings in the adiabatic potentialenergy curves (e.g., $[9,10])$. Any method for estimating MN

Published by the American Physical Society under the terms of the Creative Commons Attribution 4.0 International license. Further distribution of this work must maintain attribution to the author(s) and the published article's title, journal citation, and DOI. Funded by Bibsam. cross sections thus hinges on the ability to calculate the ioniccovalent interactions.

A promising approach is the surface integral method. The general technique was independently developed roughly simultaneously by a number of workers and thus is known by various names, including the Holstein-Herring method (e.g., [11]), the Firsov-Landau-Herring method (e.g., [12]), and the Landau-Herring method (e.g., [13-15]). The original papers on the idea include those by Firsov [16], Holstein [17], and Herring [18], and the method was used to solve problems in Landau and Lifshitz's text Quantum Mechanics [19], published in Russian in the 1950s; the asymptotic (large internuclear distance $R$ ) splitting between gerade and ungerade states for $\mathrm{H}_{2}^{+}$is solved in Sec. 81 of Ref. [19], building on problem 3 in Sec. 50. The basic idea is that the interaction energy can be calculated from the electronic current flowing across a surface between the two nuclei, obtained by manipulating Schrödinger equations corresponding to cases where the electron is localized on either nucleus. On the assumption that the wave functions are close to zero at the surface, i.e., in the limit of large internuclear separations, the method allows the interaction to be derived in terms of the atomic electronic wave functions at the surface.

A key point in the use of the method is the choice of the integration surface to ensure that wave functions are small. Smirnov [20] derived expressions for the interaction in the $\mathrm{A}^{+}+e+\mathrm{B}$ system, assuming the interaction between $e$ and $\mathrm{B}$ to be zero and taking the integration surface as the midplane, halfway between the two nuclei. Janev and Salin $[14,15]$ instead account for the potential due to $\mathrm{B}$ and chose the integration surface to be a sphere around B where the potential becomes suitably small; see also Ref. [13] for an earlier application in resonant charge transfer. 
Janev and Salin's expressions for the couplings from the surface integral method, which for consistency with previous work $[21,22]$ we will call the Landau-Herring-Janev (LHJ) method, have been used in various studies of $\mathrm{MN}$ involving $\mathrm{H}^{-}$, coupled with Landau-Zener dynamics, including $\mathrm{MN}$ of alkalis with $\mathrm{H}^{-}$[23], $\mathrm{H}_{3}^{+}$with $\mathrm{H}^{-}$[24], $\mathrm{Si}^{+}$with $\mathrm{H}^{-}$[25], and $\mathrm{H}^{+}$and $\mathrm{Be}^{+}$with $\mathrm{H}^{-}$[26]. In Refs. [21,22] the method was compared with full quantum and linear combination of atomic orbitals (LCAO) calculation results for $\mathrm{MN}$ of $\mathrm{Li}^{+}$, $\mathrm{Na}^{+}$, and $\mathrm{Mg}^{+}$with $\mathrm{H}^{-}$, and the results were found to differ significantly. Recent experimental results for branching fractions in $\mathrm{Li}^{+} / \mathrm{D}^{-}[1,2]$ and $\mathrm{Na}^{+} / \mathrm{D}^{-}$[3] have allowed calculations to be tested, and full quantum and LCAO results [7] have shown good agreement, implying the LHJ method is in disagreement with these experiments. This is puzzling, given that the LHJ method has been shown to produce estimates in reasonable agreement with experiment for $\mathrm{MN}$ of $\mathrm{N}^{+} / \mathrm{O}^{-}$and $\mathrm{O}^{+} / \mathrm{O}^{-}[27,28]$.

Asymptotic methods such as LHJ and LCAO are of significant importance for many applications, as estimates can be made rather inexpensively compared to full quantum calculation (quantum chemistry potentials and couplings with quantum scattering), and thus, it is valuable to resolve the origin of this discrepancy. The fact that the LHJ method appears to work well for $\mathrm{MN}$ involving $\mathrm{O}^{-}$and less well for the simpler $\mathrm{H}^{-}$would seem to suggest the following possible explanations: (1) there is a problem with the $\mathrm{H}^{-}$ wave function used, (2) the agreement for $\mathrm{O}^{-}$is fortuitous, or (3) the full quantum, LCAO, and experimental results are in error. In this paper it will be shown that an error appears to have been made in deriving the asymptotic $\mathrm{H}^{-}$wave function used in all applications to $\mathrm{MN}$ involving $\mathrm{H}^{-}$, and correcting this error brings the LHJ method into reasonable agreement with other theories and experiments. Previous claims that the discrepancy between the LHJ and LCAO methods is due to fundamental problems in the LCAO method are reexamined in light of this.

\section{THEORY}

Mutual neutralization occurs predominantly at avoided crossings between adiabatic molecular potential curves, corresponding to real crossings in a diabatic representation (e.g., $[9,10])$. In a diabatic representation the states of the system can be described in terms of ionic and covalent configurations. The ionic configuration is the case where the active electron is located on core $\mathrm{B}$, that is, $\mathrm{A}^{+}+(e+\mathrm{B})$, labeled $i$ with the corresponding diabatic electronic wave function $\Phi_{i}$, and the covalent configuration is the case where the active electron is located on core $\mathrm{A}^{+}$, that is, $\left(\mathrm{A}^{+}+e\right)+\mathrm{B}$, labeled $c$ with wave function $\Phi_{c}$; see Fig. 1 for a sketch of the system. These two configurations have corresponding diabatic states with potentials that cross, $H_{i i}=\left\langle\Phi_{i}|H| \Phi_{i}\right\rangle$ and $H_{c c}=\left\langle\Phi_{c}|H| \Phi_{c}\right\rangle$, where $H$ is the Hamiltonian, and have an off-diagonal interaction (coupling) $H_{i c}=\left\langle\Phi_{i}|H| \Phi_{c}\right\rangle$. In the adiabatic representation, the potentials avoid crossing, and a key quantity is the splitting between the adiabatic potential curves $\Delta U$, as it enters the Landau-Zener formula for the transition probability $[9,29,30]$. The transition probability between adiabatic states is (in atomic units, which are used

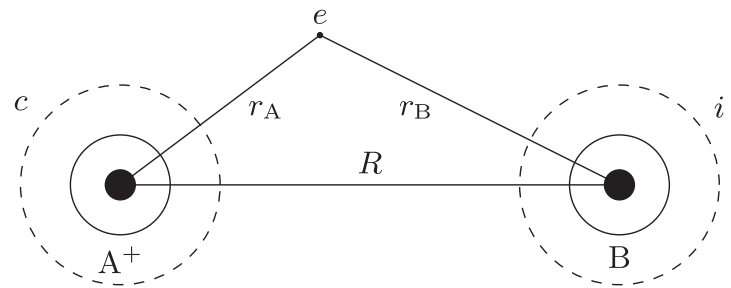

FIG. 1. Sketch of the $\mathrm{A}^{+}+e+\mathrm{B}$ system, where $\mathrm{A}^{+}$is the singly charged core, $e$ is the active electron, and $\mathrm{B}$ is a neutral atom. In the MN process [Eq. (1)], the electron moves from being localized on center $\mathrm{B}$, the ionic configuration $i$, to being localized on $\mathrm{A}$, the covalent configuration $c$.

throughout)

$$
P=\exp \left(-\frac{\pi \Delta U^{2}}{2 v_{R}\left|d\left(H_{i i}-H_{c c}\right) / d R\right|_{R=R_{x}}}\right),
$$

where $v_{R}$ is the radial component of the velocity of the relative motion of the nuclei, $R$ is the internuclear distance, and $R_{x}$ is the distance at which the diabatic potentials $H_{i i}$ and $H_{c c}$ cross. The splitting $\Delta U$ is related to the interaction $H_{i c}=\left\langle\Phi_{i}|H| \Phi_{c}\right\rangle$ in the (nonorthogonal) diabatic basis (e.g., [31,32]),

$$
\Delta U\left(R_{x}\right)=\frac{\left|\Delta\left(R_{x}\right)\right|}{1-S_{i c}^{2}}, \quad \Delta\left(R_{x}\right)=\left.2\left(H_{i i} S_{i c}-H_{i c}\right)\right|_{R=R_{x}},
$$

where $S_{i c}=\left\langle\Phi_{i} \mid \Phi_{c}\right\rangle$ is the overlap between states $i$ and $c$. Note that if the diabatic basis is orthogonal,

$$
\Delta U\left(R_{x}\right)=\Delta\left(R_{x}\right)=2 H_{i c}\left(R_{x}\right) .
$$

The references mentioned above, as well as Refs. [10,21,33], can be consulted for more details on the Landau-Zener model and the relationship between adiabatic and diabatic representations, including orthogonal and nonorthogonal representations.

\section{A. Surface integral method}

The surface integral method can be used to estimate the quantity $\Delta$ via

$$
\Delta\left(R_{x}\right)=\int_{s}\left(\Phi_{i}^{*} \nabla \Phi_{c}-\Phi_{c}^{*} \nabla \Phi_{i}\right) d \vec{s},
$$

where $s$ is the surface of integration; see Refs. [12,14,15,31] for more details. Note that here, the wave functions $\Phi$ are only the spatial components.

The LHJ theory, which chooses the surface $s$ to be a sphere centered on $B$, derives an analytic expression for $\Delta\left(R_{x}\right)$ written in terms of the asymptotic forms of the spatial atomic wave function $\varphi$ of the active electron. That is,

$$
\Phi(\vec{r}, \vec{R}) \underset{R \rightarrow \infty}{\longrightarrow} \varphi(\vec{r})=\mathcal{R}_{n l}(r) Y_{l m}(\theta, \phi),
$$

such that $\mathcal{R}_{n l}(r)$ is the radial part of the wave function, $Y_{l m}$ is the spherical harmonic function, and $\vec{r}$ is the active electron coordinate with respect to the relevant nucleus $\left(\overrightarrow{r_{A}}\right.$ or $\left.\overrightarrow{r_{B}}\right)$. When the active electron is on core $\mathrm{A}^{+}$, i.e., the system is in the covalent configuration $c$, the asymptotic behavior of the Coulomb wave function (i.e., the leading term of the 
asymptotic expansion) is used:

$$
\mathcal{R}_{c}(r)=N_{c} r^{1 / \gamma_{c}-1} \exp \left(-\gamma_{c} r\right),
$$

where $-\gamma_{c}^{2} / 2$ is the binding energy of the electron and the quantum numbers $n l$ are now dropped from the radial function $\mathcal{R}$ for simplicity. When the electron is on core $\mathrm{B}$, thus forming a negative ion, and the system is in the ionic configuration $i$, the appropriate asymptotic form is used:

$$
\mathcal{R}_{i}(r)=N_{i} \frac{2 \gamma_{i}}{\pi} k_{l_{i}}\left(\gamma_{i} r\right),
$$

where $-\gamma_{i}^{2} / 2$ is the binding energy of the electron, and thus, $E_{\mathrm{B}}^{-}=\gamma_{i}^{2} / 2$ is the electron affinity of the negative ion, $k_{l}(x)$ is the modified spherical Bessel function (e.g., [34]), and $l_{i}$ is the orbital angular momentum quantum number. For the specific case of $l_{i}=0$,

$$
\mathcal{R}_{i}(r)=N_{i} \exp \left(-\gamma_{i} r\right) / r .
$$

Both wave functions contain leading constants, $N_{c}$ and $N_{i}$, that must be determined. In the case of $N_{c}$, it can be estimated from the Coulomb wave-function normalization constant from quantum defect theory [35-37] (see, e.g., Eq. (19) of Ref. [14]), which can be expected to give accurate results for sufficiently excited states with diffuse wave functions well described by quantum defect theory. The leading-constant factor for the negative-ion function is, however, more problematic to determine. Due to the $1 / r$ factor, the normalization is dominated by contributions at short distances where the asymptotic form is not valid and overestimates the wave function, and the value given by normalization $N_{i}=\sqrt{2 \gamma_{i}}$ (see Ref. [38]) cannot be expected to give an accurate representation of the asymptotic behavior. $N_{i}$ should therefore be determined by comparison with detailed atomic structure calculations. One expects $N_{i}>\sqrt{2 \gamma_{i}}$, and this has sometimes led to the use of a cutoff value $r_{0}$, below which the wave function is zero and can be chosen to give correct normalization (e.g., Refs. [21,38]).

Using these wave functions, Refs. $[15,39]$ give the expression for $\Delta\left(R_{x}\right)$ as

$$
\begin{aligned}
\Delta\left(R_{x}\right)= & \frac{\delta_{m_{i} m_{c}} N_{i} \mathcal{R}_{c}\left(R_{x}\right)}{\left(2 \gamma_{i} R_{x}\right)^{\left|m_{c}\right|}\left|m_{c}\right| !} \\
& \times\left[\frac{\left(2 l_{c}+1\right)\left(2 l_{i}+1\right)\left(l_{c}+\left|m_{c}\right|\right) !\left(l_{i}+\left|m_{i}\right|\right) !}{\left(l_{c}-\left|m_{c}\right|\right) !\left(l_{i}-\left|m_{i}\right|\right) !}\right]^{1 / 2},
\end{aligned}
$$

which for $l_{i}=0$, as in the case of $\mathrm{H}^{-}\left({ }^{1} S\right)$, reduces to the rather simple expression

$$
\Delta\left(R_{x}\right)=N_{i} \mathcal{R}_{c}\left(R_{x}\right) \times\left(2 l_{c}+1\right)^{1 / 2} .
$$

The modification due to angular momentum coupling to core electrons in complex systems, including those with equivalent electrons, is given in Refs. [12,15,39]. The major advantage of this approach is that, as can be seen, it reduces to an analytic expression and does not require the evaluation of any matrix elements of the Hamiltonian.

\section{B. LCAO method}

The LCAO approach can be used to calculate $\Delta\left(R_{x}\right)$ through the calculation of the matrix elements of the
Hamiltonian using the same or similar atomic wave functions for the active electron. This was done in Refs. [21,32,40,41] for the case involving $\mathrm{H}^{-}$, considering two electrons. The main differences between these different descriptions are the treatment of the two-electron wave functions, leading to slightly different expressions, but they are equivalent asymptotically (at large separations $R$ ). For the purposes of our discussion, a much simpler one-electron version is preferable, again equivalent to the above formulations at large $R$.

Considering the model and coordinate system given in Fig. 1, ignoring any interactions with the neutral atom $\mathrm{B}$, the Hamiltonian is $H=-1 / r_{A}$, and we have the rather simple expression in terms of atomic wave functions

$$
H_{i i}=\left\langle\varphi_{i}\left|-\frac{1}{r_{A}}\right| \varphi_{i}\right\rangle,
$$

where for large $R$ we have

$$
\left\langle\varphi_{i}\left|\frac{1}{r_{A}}\right| \varphi_{i}\right\rangle \approx \frac{1}{R},
$$

and similarly,

$$
H_{i c}=\left\langle\varphi_{i}\left|-\frac{1}{r_{A}}\right| \varphi_{c}\right\rangle .
$$

Using these results in Eq. (3), we obtain

$$
\begin{aligned}
\Delta\left(R_{x}\right) & \left.\approx 2\left(-\frac{1}{R}\left\langle\varphi_{i} \mid \varphi_{c}\right\rangle-\left\langle\varphi_{i}\left|-\frac{1}{r_{A}}\right| \varphi_{c}\right\rangle\right)\right|_{R=R_{x}} \\
& \left.\approx 2\left\langle\varphi_{i}\left|\frac{1}{r_{A}}-\frac{1}{R}\right| \varphi_{c}\right\rangle\right|_{R=R_{x}},
\end{aligned}
$$

where we now define

$$
T_{i c}(R) \equiv 2\left\langle\varphi_{i}\left|\frac{1}{r_{A}}-\frac{1}{R}\right| \varphi_{c}\right\rangle,
$$

the "postinteraction operator" $T$ of charge-transfer theory also found in Refs. [32,40]. The same result is found through a different derivation in Sec. 10.3 of Ref. [42].

Analytic expressions for $T_{i c}(R)$ can be obtained by adopting the asymptotic forms of the atomic wave functions given above, although for any given $l_{c}$ and $l_{i}$, they are significantly more complex than those arising from the surface integral method for $\Delta\left(R_{x}\right)$ given above. Expressions for both $T_{i c}(R)$ and $S_{i c}(R)$ for the $l_{c}=l_{i}=0$ case are given in the Appendix.

\section{C. $\mathrm{H}^{-}$asymptotic wave function}

The general theory of asymptotic wave functions has been well expounded, for example, in Sec. 2.1 of Ref. [43], as well as Ref. [44]. The derivation of the asymptotic wave function for $\mathrm{H}^{-}$is particularly simple, and so it is instructive for the following discussion to present the basic equations (see, e.g., Refs. $[41,42,44]$ for details). The two-electron nonrelativistic Hamiltonian, in atomic units, is

$$
H=-\frac{1}{2} \nabla_{1}^{2}-\frac{1}{2} \nabla_{2}^{2}-\frac{1}{r_{1}}-\frac{1}{r_{2}}+\frac{1}{r_{12}},
$$

where $r_{1}$ and $r_{2}$ are distances of electrons with labels 1 and 2 to the proton and $r_{12}$ is the distance between electrons. Taking the asymptotic case $r_{1} \gg r_{2}$, then $r_{12} \approx r_{1}$, the asymptotic form then allows separation into terms related to each 
electron,

$$
H \approx\left(-\frac{1}{2} \nabla_{1}^{2}\right)+\left(-\frac{1}{2} \nabla_{2}^{2}-\frac{1}{r_{2}}\right) \equiv H_{1}+H_{2},
$$

such that $H_{1}$ is the Hamiltonian for the loosely bound distant electron and $\mathrm{H}_{2}$ is the Hamiltonian for the tightly bound electron close to the proton. Then the time-independent Schrödinger equation becomes

$$
\left(H_{1}+H_{2}\right) \psi=\left(-E_{\mathrm{H}^{-}}+E_{\mathrm{H}}^{1 s}\right) \psi,
$$

where $\psi$ is the electronic wave function, $E_{\mathrm{H}}^{1 s}$ is the energy of the hydrogen ground state $(-0.5$ a.u. $)$, and $E_{\mathrm{H}^{-}}$is the electron affinity of $\mathrm{H}$ (a positive value; see below). Separating the total wave function $\psi$ into spatial and spin functions $\psi=\varphi\left(\vec{r}_{1}, \vec{r}_{2}\right) \chi_{S=0}$, where $\chi_{S=0}$ is the singlet spin function, the asymptotic spatial wave function becomes

$$
\varphi^{\text {asymp }}\left(\vec{r}_{1}, \vec{r}_{2}\right)=\varphi_{\mathrm{LR}}\left(\vec{r}_{1}\right) \varphi_{1 s}^{\mathrm{H}}\left(\vec{r}_{2}\right),
$$

where $H_{2} \varphi_{1 s}^{\mathrm{H}}\left(\vec{r}_{2}\right)=E_{\mathrm{H}}^{1 s} \varphi_{1 s}^{\mathrm{H}}\left(\vec{r}_{2}\right) \quad$ and thus $\varphi_{1 s}^{\mathrm{H}}(\vec{r})=$ $\exp (-r) / \sqrt{\pi}$ is the hydrogen ground-state function, and the long-range (LR) electron function is the solution of

$$
H_{1} \varphi_{\mathrm{LR}}\left(\vec{r}_{1}\right)=\left(-\frac{1}{2} \nabla_{1}^{2}\right) \varphi_{\mathrm{LR}}\left(\vec{r}_{1}\right)=-E_{\mathrm{H}^{-}} \varphi_{\mathrm{LR}}\left(\vec{r}_{1}\right) .
$$

The spherically symmetric $(l=0)$ solution is

$$
\varphi_{\mathrm{LR}}(\vec{r})=A \exp (-\gamma r) / r \quad \gamma r \gg 1,
$$

where $\gamma=\sqrt{2 E_{\mathrm{H}^{-}}}$and $A$ is an arbitrary constant related to $N_{i}$ in Eq. (9), which we need to determine. The electron affinity of $\mathrm{H}$ from modern measurements is $E_{\mathrm{H}^{-}}=0.754195(19) \mathrm{eV}$ or 0.0277162 a.u. [45], which gives $\gamma=0.235441$ in atomic units. The validity condition $\gamma r \gg 1$ thus requires $r \gg 1 / \gamma=$ 4.26 a.u. As will be shown below, $A=N_{i} \frac{1}{\sqrt{4 \pi}}$, and correct normalization would imply $N_{i}=\sqrt{2 \gamma} \approx 0.686$ and $A \approx 0.194$.

Unfortunately, a range of different definitions and notations for the asymptotic function and the leading constant have been used in various sources. Part of this difference often arises from separation into radial and angular components, for this case $(l=0)$

$$
\varphi_{\mathrm{LR}}(\vec{r})=\mathcal{R}(r) Y_{00}(\theta, \phi)=\mathcal{R}(r) \frac{1}{\sqrt{4 \pi}} .
$$

In early papers by Smirnov [20], the following definition was used:

$$
\mathcal{R}(r)=A_{\mathrm{S} 65} \sqrt{2 \gamma} \exp (-\gamma r) / r,
$$

where $\sqrt{2 \gamma}$ arises because the function is correctly normalized if $A_{\mathrm{S} 65}=1$ (see Sec. II A). In Ref. [20], the value $A_{\mathrm{S} 65}^{2}=$ 2.65 was derived, which means $A_{\mathrm{S} 65}=1.63$. This implies $A=A_{\mathrm{S} 65} \sqrt{2 \gamma} \frac{1}{\sqrt{4 \pi}}=0.316$. Later work by Smirnov and others $[14,23,38,42,46]$ adopted the definition

$$
\mathcal{R}(r)=A_{\mathrm{S}} \exp (-\gamma r) / r=B_{\mathrm{S}} \sqrt{2 \gamma} \exp (-\gamma r) / r,
$$

such that $B$ in Ref. [42], here denoted $B_{S}$, is equal to $A_{\mathrm{S} 65}$. Note that $A_{S}=N_{i}$ and is thus the value required for use in the equations of Janev and Salin [14,15], that is, Eqs. (10) and (11). Smirnov $[38,42,46]$ derived $A_{S}$ from the simple Chandrasekhar two- and three-parameter wave functions [47], finding a value of $A_{\mathrm{S}}=1.13 \pm 0.06$, and thus, $B_{\mathrm{S}}=A_{\mathrm{S} 65}=$ $1.64 \pm 0.08$. A value of $A_{S}$ in this range has been used in all applications of the LHJ method to processes involving $\mathrm{H}^{-}$ [21-26]. This gives $A=A_{\mathrm{S}} \frac{1}{\sqrt{4 \pi}}=0.319$.

In LCAO applications [40,41] the value $A=0.223106$ has been used, determined from the 203-parameter variational wave function of Pekeris [48], with asymptotic values of the wave function being presented in Ref. [49]. This derivation will be discussed in Sec. II C 1. In Ref. [22] the LCAO method was applied, and the same value is essentially used, but a factor of $\sqrt{2}$ must be applied to adjust for the antisymmetrized version of the asymptotic wave function that was employed.

Clearly, there is a discrepancy between the leading constant of the asymptotic wave function derived by Smirnov and used in all LHJ theory applications, $A \approx 0.319$, and the value that has been applied in LCAO calculations, i.e., $A \approx 0.223$. The two values of $A$ differ by a factor of roughly $\sqrt{2}$. In the following we repeat both derivations in detail to show the source of the problem.

\section{Direct matching of the asymptotic wave function}

The most straightforward approach to determining the appropriate constant in the asymptotic wave function is through direct matching to a calculated wave function for $\mathrm{H}^{-}$. This is the approach that has been used in the LCAO case, and the method stems from Ref. [49]. They defined a function equal to the two-electron spatial wave function where either $r_{1}=0$ or $r_{2}=0, \psi(r)$ in their notation. Here, we denote this same function:

$$
\begin{aligned}
\varphi_{0}(r) & \equiv \varphi\left(\vec{r}_{1}=0,\left|\vec{r}_{2}\right|=r\right)=\varphi\left(\left|\vec{r}_{1}\right|=r, \vec{r}_{2}=0\right) \\
& =C(r) \exp (-\gamma r) / r,
\end{aligned}
$$

such that $C(r)$ is the same as in Ref. [49] and converges to a constant value $C(\infty)$ at large $r$ [50]. Equating to the asymptotic wave function from above, Eq. (20), taking $r_{2}=0$, we obtain

$$
\begin{aligned}
\varphi_{0}^{\text {asymp }}(r) & =\varphi_{\mathrm{LR}}\left(\left|\vec{r}_{1}\right|=r\right) \varphi_{1 s}^{H}\left(\overrightarrow{r_{2}}=0\right) \\
& =\varphi_{\mathrm{LR}}(r) \frac{1}{\sqrt{\pi}} \\
& =A \exp (-\gamma r) / r \frac{1}{\sqrt{\pi}},
\end{aligned}
$$

which means $A=C(\infty) \sqrt{\pi}$. In Ref. [49], $C(\infty)=0.125874$, and thus, $A=0.223106$ was found by matching points at $r=$ $14-16$ a.u. from the calculated values of $\varphi_{0}(r)$ extracted from the 203-parameter wave function of Ref. [48] given in their Table 1. This is the value used in LCAO calculations.

Figure 2 compares $\varphi_{0}(r)$ extracted from various detailed calculations of the wave function $\varphi\left(\vec{r}_{1}, \vec{r}_{2}\right)$ with asymptotic forms of the wave function $\varphi_{0}^{\text {asymp }}(r)$ for the two different values of $A$ discussed above. It is clearly seen that $A \sim 0.223$ matches all the functions better than the higher value of $A \sim$ 0.319 . Note that among detailed calculations, only the Pekeris wave function has the expected asymptotic form even at very long range since the variational form of the wave function used ensures correct asymptotic behavior. The two- and threeparameter wave functions derived by Chandrasekhar [47] are of particular interest as they were used by Smirnov and also because they are so simple that they can be used to exemplify 


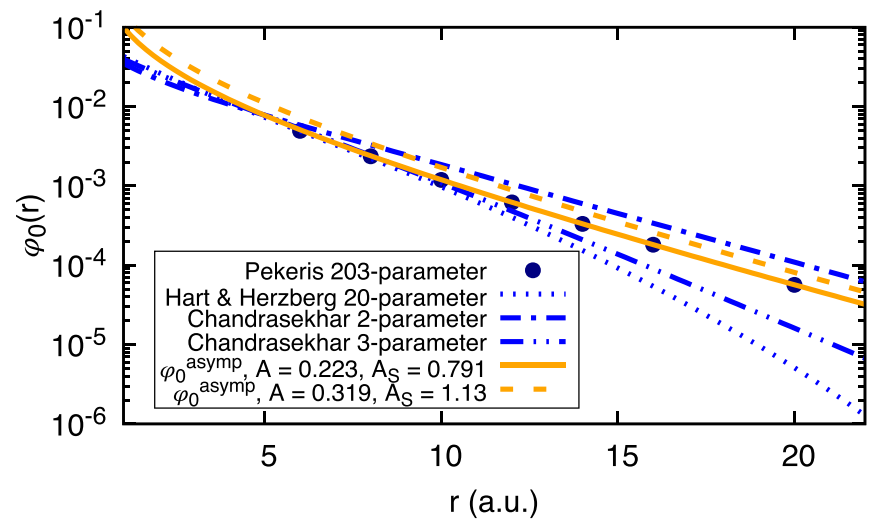

FIG. 2. The asymptotic $\mathrm{H}^{-}$wave function $\varphi_{0}(r)$ as defined in Eq. (26), extracted from various detailed calculations of the wave function $\varphi\left(\vec{r}_{1}, \vec{r}_{2}\right)$ : the 203-parameter function from Pekeris [48] calculated in Ref. [49], the 19-parameter function of Hart and Herzberg [51], and the 2- and 3-parameter functions of Chandrasekhar [47]. Asymptotic forms [Eq. (27)] with the two values of $A$ discussed in the text are also shown.

various issues. The Chandrasekhar wave function is

$$
\begin{aligned}
\varphi\left(\vec{r}_{1}, \vec{r}_{2}\right)= & N\left[\exp \left(-a r_{1}-b r_{2}\right)+\exp \left(-a r_{2}-b r_{1}\right)\right] \\
& \times\left(1+c r_{12}\right),
\end{aligned}
$$

where for the two-parameter function $(c=0)$ he found through variational calculations $a=1.03925$ and $b=$ 0.28309 and it can be found from the normalization condition that $N=0.031443$ and for the three-parameter function introducing correlation he found $a=1.07478, b=0.47758$, $c=0.31214$ and it can be found that $N=0.031226$. That $a \approx$ 1 in both cases shows the expected result of an unscreened hydrogen $1 s$-like function for the electron close to the proton, with a more distant long-range electron. Having said that, it is clear that the functions do not have the correct asymptotic behavior when the distant electron is very far from the nucleus. The Chandrasekhar 2-parameter function does not have asymptotic behavior at any distance, and the 3-parameter and Hart and Herzberg 20-parameter functions [51] have only roughly asymptotic behavior in the region $r \approx 4-10 \mathrm{a}$.u. This is due to the fact that the asymptotic region has little influence on the state energies and thus the wave function determined by variational methods.

\section{Electronic-density-matching method}

An alternative approach, which has been used by Smirnov $[38,42,46]$, is to match the electron density function. Generally, for an $N$-electron system, ignoring spin, the electronic density is

$$
\rho(\vec{r})=N \int d \overrightarrow{r_{2}} \cdots \int d \overrightarrow{r_{N}}\left|\varphi\left(\vec{r}, \overrightarrow{r_{2}}, \ldots, \overrightarrow{r_{N}}\right)\right|^{2},
$$

and thus, for $\mathrm{H}^{-}$and two electrons

$$
\rho(\vec{r})=2 \int d \vec{r}_{2}\left|\varphi\left(\vec{r}, \overrightarrow{r_{2}}\right)\right|^{2}
$$

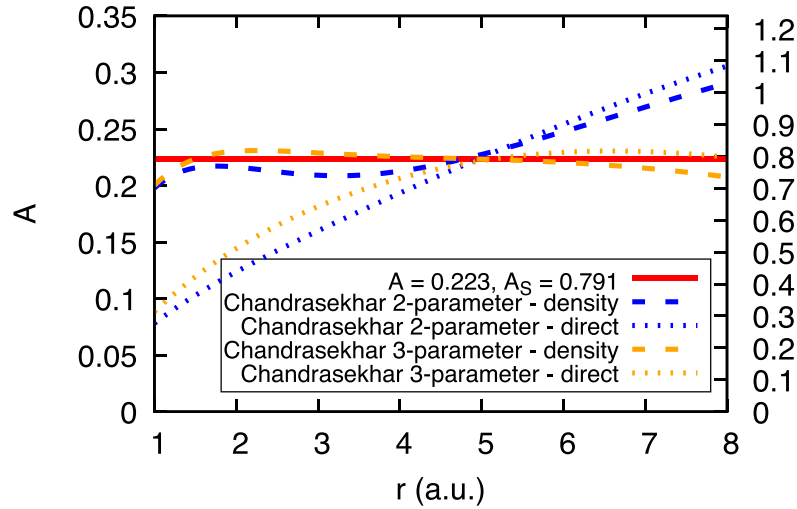

\section{$\stackrel{\infty}{\leftarrow}$}

FIG. 3. Derived values for $A$ as a function of $r$. Corresponding values for $A_{\mathrm{S}}$ are shown on the right axis. The blue (dark gray) lines show the values derived from Chandrasekhar's two-parameter wave function, and the orange (light gray) lines are from the threeparameter wave function. In each case, dashed lines are from the electronic density matching method, and dotted lines are from the direct matching method. The horizontal solid red line shows the value derived from direct matching to the accurate wave function due to Pekeris [48]; see Fig. 2 and Sec. II C 1.

For the asymptotic form this gives

$$
\begin{aligned}
\rho^{\operatorname{asymp}}(\vec{r}) & =2 \int d \overrightarrow{r_{2}}\left|\varphi_{\mathrm{LR}}(\vec{r}) \varphi_{1 s}^{H}\left(\overrightarrow{r_{2}}\right)\right|^{2} \\
& =2\left|\varphi_{\mathrm{LR}}(\vec{r})\right|^{2} \\
& =2 A^{2} \exp (-2 \gamma r) / r .
\end{aligned}
$$

As done by Smirnov, one can derive analytical expressions for the asymptotic constant $A$ or some related version of it, e.g., $A_{\mathrm{S}}$ or $A_{\mathrm{S} 65}$, as a function of $r$ by equating the asymptotic form with the electronic density calculated from Chandrasekhar's wave functions, and the expressions found by Smirnov are given in Refs. [38,42]. Using Mathematica, this analysis was repeated here, making use of Hylleraas coordinates for the three-parameter case [52,53], and the results are plotted in Fig. 3.

From Fig. 3 it can be seen that the values of $A$ and $A_{S}$ as a function of $r$ from electronic density matching of both the two- and three-parameter Chandrasekhar wave functions are roughly constant and in agreement around $r=2-5$ a.u., the region used by Smirnov to derive $A_{S}$. Figure 3 clearly favors a low value, $A \approx 0.22\left(A_{S} \approx 0.79\right)$, rather than a high value, $A \approx 0.32\left(A_{S} \approx 1.13\right)$. Thus, the analysis presented here from electron density matching is roughly in agreement with the value from direct matching, $A=0.223\left(A_{S}=0.791\right)$, which is roughly a factor of $1 / \sqrt{2}$ lower than that found by Smirnov, $A \approx 0.32\left(A_{S} \approx 1.13\right)$. The difference is not precisely a factor of $1 / \sqrt{2}$ since different wave functions are used, the direct method permitting the easy use of the accurate 203-parameter function of Pekeris.

Note that the criterion for validity of the asymptotic wave function, $r \gg 1 / \gamma=4.26$ a.u., is not satisfied across this entire region $r=2-5$ a.u. Further, it is clear that the Chandrasekhar wave functions do not have correct asymptotic behavior, as is seen from application of the direct method, which gives rapidly varying values of $A$ (see also Fig. 3 ). That 
the density-matching method using these simple wave functions gives values that agree reasonably with the values from direct matching of the accurate and asymptotically correct wave function of Pekeris is thus perhaps somewhat surprising. It may reflect that the density method, by accounting for contributions from both electrons at intermediate distances, reaches asymptotic behavior at smaller internuclear distances than the direct method, in which the second electron is fixed at the nucleus. Contributions from both electrons to the wave function are equal, $\varphi_{\mathrm{LR}}(\vec{r})=\varphi_{1 s}^{H}(\vec{r})$, at roughly $r=2.3$ a.u.

\section{COMPARISON OF THEORETICAL CALCULATIONS AND EXPERIMENTS}

Experiments were recently performed in Louvain on $\mathrm{MN}$ of $\mathrm{Li}^{+} / \mathrm{D}^{-}$[1] and at the Double ElectroStatic Ion Ring ExpEriment (DESIREE) facility in Stockholm on $\mathrm{Li}^{+} / \mathrm{D}^{-}$ [2] and $\mathrm{Na}^{+} / \mathrm{D}^{-}$[3], resolving final states and thus measuring branching fractions for the neutral products. $\mathrm{D}^{-}$is preferred over $\mathrm{H}^{-}$in the experiments for practical reasons but is basically identical to $\mathrm{H}^{-}$in terms of binding energy (electron affinity) and thus electronic structure. The different mass leads to trajectory (Coulomb-focusing) effects but is easily accounted for in the dynamical calculations. In a recent paper [7], existing full quantum calculations [54-56] and (two-electron) LCAO method calculations [21,22] were compared with experiment, generally finding good agreement, with the full quantum calculations performing best, in line with expectations. The reader is referred to Ref. [7] for a discussion regarding these comparisons, including the discussion of Coulomb-focusing effects caused by $\mathrm{H}^{-}$versus $\mathrm{D}^{-}$.

Here, these comparisons are supplemented with calculations from the LHJ theory using the two $A$ values discussed, and these calculations use the same code as the LCAO calculations, described in Refs. [21,22]. The results are shown for $\mathrm{Li}^{+} / \mathrm{D}^{-}$in Fig. 4 and for $\mathrm{Na}^{+} / \mathrm{D}^{-}$in Fig. 5. To aid comparison (see Ref. [7]), the plots are presented on the reduced energy scale, which is defined as $E_{\mathrm{R}}=E_{\mathrm{CM}} / \mu=\frac{1}{2} v^{2}$, where $E_{\mathrm{CM}}$ is the collision energy in the center-of-mass frame, $\mu$ is the reduced mass of the system, and $v$ is the relative velocity. It is clear that the comparisons with experiment are significantly improved for both $\mathrm{Li}^{+} / \mathrm{D}^{-}$and $\mathrm{Na}^{+} / \mathrm{D}^{-}$if the low value of $A=0.223$ is adopted, compared to the high value of $A=0.319$. In particular we note that for $\mathrm{Li}^{+} / \mathrm{D}^{-}$(Fig. 4), the high value leads to the prediction of $3 p$ being the dominant channel at low energy, rather than $3 s$, as clearly seen to a high degree of certainty in experiments. This discrepancy is resolved for the LHJ theory with the lower value of $A$. Further, the low value of $A$ leads to much better consistency between full quantum, LCAO, and LHJ predictions in both cases. Note also that LHJ with the high value of $A$ leads to variation of the branching fractions at lower collision energies.

Janev and Radulovic [23] also performed calculations with the LHJ method for $\mathrm{Li}^{+} / \mathrm{H}^{-}$and $\mathrm{Na}^{+} / \mathrm{H}^{-}$, although stateresolved results were shown for only $\mathrm{Na}^{+} / \mathrm{H}^{-}$(their Fig. 1). Our results for $\mathrm{Na}^{+} / \mathrm{H}^{-}$partial cross sections are significantly different from theirs; in particular at low energy they found $3 p$ to be the second most populated channel, only an order of magnitude lower than $4 s$. This is in strong disagreement with experiment, where the $3 p$ channel is not observed, and
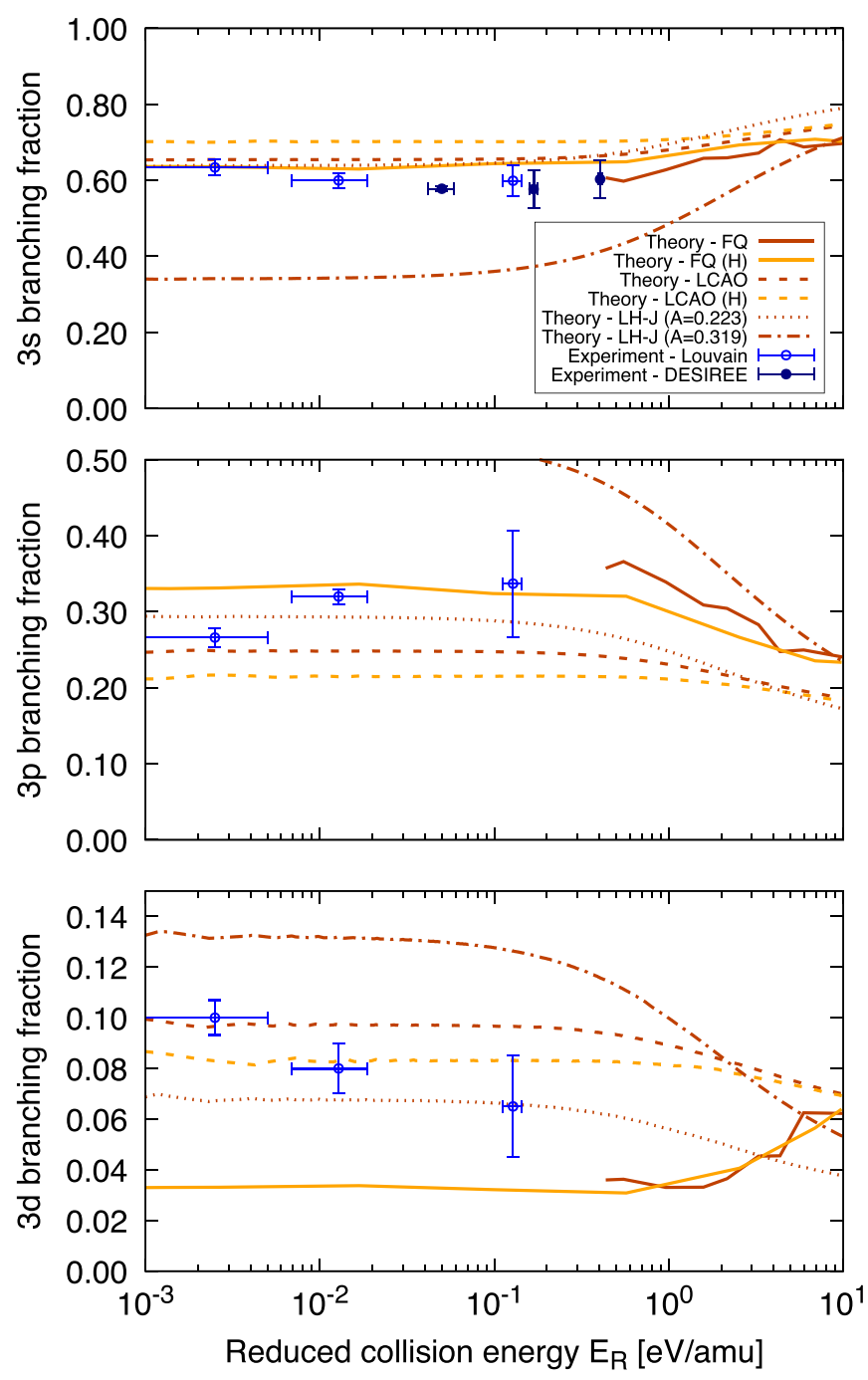

FIG. 4. Branching fractions for the $\mathrm{MN}$ reaction $\mathrm{Li}^{+}+\mathrm{D}^{-} \rightarrow$ $\mathrm{Li}(n l)+\mathrm{D}$ as a function of reduced collision energy. The $3 s, 3 p$, and $3 d$ channels are shown in separate panels. Experimental results from Louvain [1] and from DESIREE [2] are shown, with estimated errors $(1 \sigma)$. Theoretical results are shown from full quantum (FQ) calculations and LCAO and LHJ asymptotic methods with the old (high) value for $A$ and the new (low) value for $A$; see text for further details. Theoretical results are also shown for the case where $\mathrm{D}^{-}$is replaced by $\mathrm{H}^{-}$, marked by $(\mathrm{H})$ in the legend.

with all other calculations, including our LHJ calculations with the old, high value of $A$, for which we find the cross section for $3 p$ to be more than five orders of magnitude lower than $4 s$; this may suggest a labeling error. In Ref. [56] substantial differences were also noted compared to full quantum calculations. This was investigated further by comparing our LHJ theory avoided-crossing parameters with those in Table 1 of Ref. [23], in particular $\Delta\left(R_{x}\right)$. However, for both Li and $\mathrm{Na}$ we were unable to reproduce all of their results using the equations and numbers in the paper. Using their equations, their value for the leading constant in the negative-ion radial wave function, and their values for $R_{x}$, we obtain $\Delta\left(R_{x}\right)$ in agreement (within 10\%) for a few crossings ( $\mathrm{Li} 2 s$ and $2 p$, $\mathrm{Na} 3 p$ ), but for the majority there are significant differences 

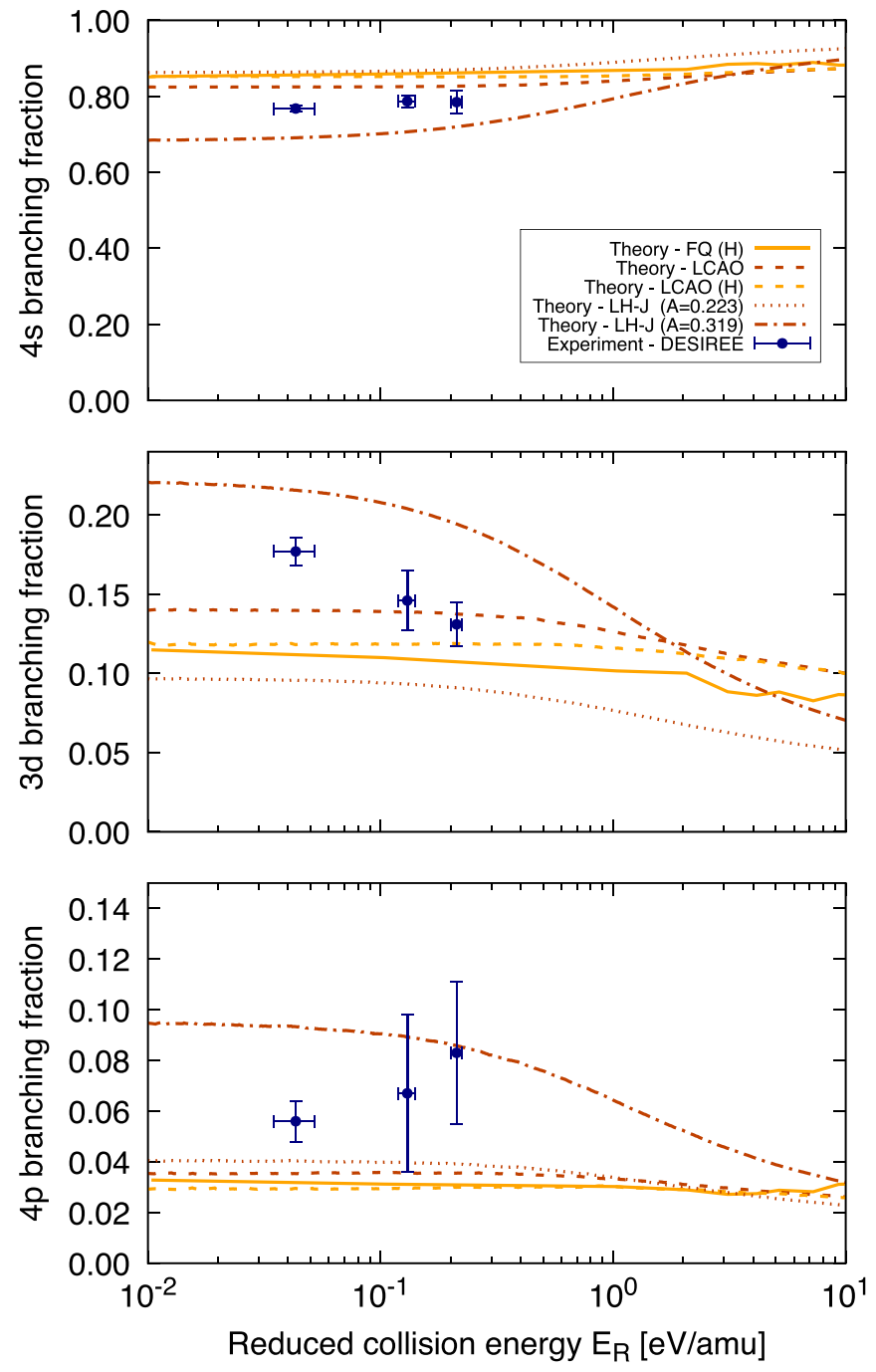

FIG. 5. Branching fractions for the MN reaction $\mathrm{Na}^{+}+\mathrm{D}^{-} \rightarrow$ $\mathrm{Na}(n l)+\mathrm{D}$ as a function of reduced collision energy. The $4 s, 3 d$, and $4 p$ channels are shown in separate panels. Experimental results from DESIREE [3] are shown, with estimated errors $(1 \sigma)$. Theoretical results are shown from full quantum (FQ) calculations and LCAO and LHJ asymptotic methods with the old (high) value for $A$ and the new (low) value for $A$; see text for further details. Theoretical results are also shown for the case where $\mathrm{D}^{-}$is replaced by $\mathrm{H}^{-}$, marked by $(\mathrm{H})$ in the legend.

(up to a factor of 2.5 for $\mathrm{Na} 4 p$ ), with our result usually being larger (only for $\mathrm{Li} 3 d$ do we obtain a roughly $20 \%$ smaller value). Our LHJ theory values using $A=0.319$ agree well with what we calculate from the equations in Ref. [23], which are just simpler versions of the more general theory. As such, detailed comparison of the avoided-crossing parameters is not particularly enlightening for the more general problem of interest here, namely, the relationship between LHJ theory, LCAO theory, and experiment.

Dochain and Urbain [57] semiempirically found a similar need for a factor of $1 / \sqrt{2}$ correction to the couplings in LHJ theory and in doing so obtained good matches to experimental results for $\mathrm{MN}$ of $\mathrm{D}^{-}$with $\mathrm{He}^{+}, \mathrm{Li}^{+}, \mathrm{Na}^{+}, \mathrm{C}^{+}$, and $\mathrm{N}^{+}$, although with some discrepancies for $\mathrm{O}^{+}$.
Finally, it is worth noting that calculations with the Smirnov formulation of the surface integral method [20], taking the surface of integration as the midplane between the two atoms, while improving with the lower value of $A$, still perform substantially worse than the Janev and Salin formulation (see also Ref. [26]).

\section{DISCUSSION}

It was claimed in Ref. [39] that the LCAO method is "essentially incorrect for the problem of calculation of $\Delta(R)$," with reference to the work of Herring [18]. It is, however, unclear that the criticisms of the LCAO method for the cases discussed by Herring, the exchange interaction [58] in $\mathrm{H}_{2}^{+}$and $\mathrm{H}_{2}$, apply also to ionic-covalent interactions. First, the problem of the $1 / r_{12}$ term described for the exchange interaction in $\mathrm{H}_{2}$ is not relevant, as it does not enter the one-electron LCAO method (see Sec. II B) and in the two-electron methods enters only empirically via the $\mathrm{H}^{-}$electron affinity and wave function (e.g., Eq. (10) of Ref. [21]). Second, in both cases, a basis with a single tightly bound $1 s$ wave function is used in the LCAO description. In the case studied in detail by Herring for the exchange splitting in $\mathrm{H}_{2}^{+}$, the surface integral approach permits a modification factor which increases the value of the wave function between the two nuclei, capturing departures of the molecular wave function from the atomic ones, which cannot be modeled in a LCAO basis with a single fixed orbital. In contrast, the LCAO method for ionic-covalent interactions employs two wave functions that capture the main components of the molecular wave function at internuclear distances corresponding to the crossing.

More quantitatively, Ref. [39] claimed that there is a discrepancy between the asymptotic behavior of the interactions in the LHJ and LCAO formulations. For LHJ the interaction has the asymptotic behavior

$$
\Delta_{\mathrm{LHJ}}\left(R_{x}\right) \propto \exp \left(-\gamma_{c} R_{x}\right)
$$

at large $R_{x}$ [see Eqs. (10) and (7)], while for LCAO at large $R$, Ref. [39] claimed the asymptotic behavior is

$$
\Delta_{\mathrm{LCAO}}\left(R_{x}\right) \propto \exp \left[-\left(\gamma_{i}+\gamma_{c}\right) R_{x}\right]
$$

however, this relationship is asserted without proof and misses the important fact that $\gamma_{i}$ and $\gamma_{c}$ are related at the crossing point $R_{x}$. Assuming a pure Coulomb interaction for the ionic potential and a null potential for the covalent state, the crossing point is given by

$$
\frac{1}{R_{x}}=\frac{\gamma_{c}^{2}}{2}-\frac{\gamma_{i}^{2}}{2} .
$$

Using the one-electron LCAO expression for $T_{i c}(R)$ for $l_{c}=l_{i}=0$ given in the Appendix and making the substitution $\gamma_{i} \rightarrow \sqrt{\gamma_{c}^{2} / 2-2 / R_{x}}$, an analytic expression for $\Delta_{\mathrm{LCAO}}\left(R_{x}\right)=T_{i c}\left(R=R_{x}\right)$ can be obtained. This results in a complicated expression which is no longer dependent on $\gamma_{i}$ and which can be shown to have the same behavior as the LHJ theory for large $R_{x}$; i.e., for $l_{c}=0$

$$
\Delta_{\mathrm{LHJ}}\left(R_{x}\right)=N_{i} \mathcal{R}_{c}\left(R_{x}\right) .
$$

This agreement is most convincingly shown by numerical results for the ratio $\Delta_{\mathrm{LCAO}} / \Delta_{\mathrm{LHJ}}$, which is dependent only on 


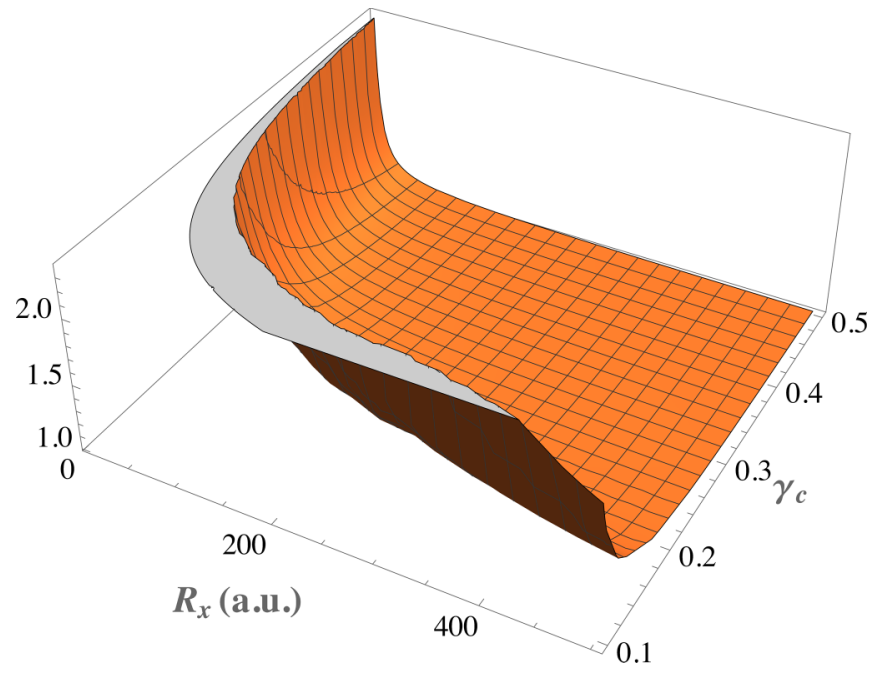

FIG. 6. Three-dimensional mesh plot of the ratio $\Delta_{\mathrm{LCAO}} / \Delta_{\mathrm{LHJ}}$ as a function of $\gamma_{c}$ and $R_{x}$ for $l_{c}=l_{i}=0$ analytic expressions obtained as described in the text. The ratio tends to 1 for $R_{x} \gg \gamma_{c}^{-2}$ (see text). The plot ranges from $n^{*}=2\left(\gamma_{c}=0.5\right)$ to $n^{*}=10\left(\gamma_{c}=0.1\right)$.

$\gamma_{c}$ and $R_{x}$, as shown in Fig. 6. The condition for validity of the LHJ method is $R_{x} \gg \gamma_{i}^{-2}$ (Ref. [14]), which, noting that for large $R_{x}$ we have $\gamma_{i} \approx \gamma_{c}-1 /\left(\gamma_{c} R_{x}\right)$, is roughly equivalent to $R_{x} \gg \gamma_{c}^{-2}$. The ratio is seen to be exactly 1 in the region of validity of the LHJ theory. Note the effective principle quantum number of the neutral state on $\mathrm{A}$ is $n^{*}=1 / \gamma_{c}$; thus, the validity criterion can also be written $R_{x} \gg n^{* 2}$. Outside the region of validity for the LHJ theory, at crossings at shorter internuclear distances, the LCAO theory gives larger interactions, and the disagreement increases very rapidly.

In summary, previous claims that LCAO theory is incorrect for ionic-covalent interactions at large internuclear distance seem to be unfounded, and correction of the leading constant for the $\mathrm{H}^{-}$asymptotic wave function in the LHJ theory brings the two theories into reasonable quantitative agreement for the two cases of $\mathrm{MN}$ of $\mathrm{Li}^{+}$and $\mathrm{Na}^{+}$with $\mathrm{H}^{-}$and $\mathrm{D}^{-}$. It has also been shown that the LCAO and LHJ theories have the same behavior for large $R_{x}$. The LCAO theory has been developed only for cases involving $\mathrm{H}^{-}$and $\mathrm{D}^{-}$, treating two electrons to varying degrees of complexity. The surface integral method, LHJ, is, however, easily applied to any given situation if the asymptotic wave function for the negative ion is known. The results shown here suggest that if correct wave functions for the active electron on the negative ion can be obtained, LHJ theory can be used to obtain reasonable estimates for MN processes occurring at a large internuclear distance in practically any system, perhaps even those involving molecules, and thus is of potentially great utility for situations such as astrophysical modeling, where often a large number of rate estimates are needed and completeness can be more important than accuracy. It would be useful also to further develop and investigate the utility of the one-electron LCAO method as outlined in this paper, enabling application to complex atoms beyond $\mathrm{H}^{-}$and $\mathrm{D}^{-}$.

\section{ACKNOWLEDGMENTS}

I thank X. Urbain, A. Dochain, J. Grumer, A. Amarsi, and G. Eklund for useful discussions, sharing preliminary results, and comments on the manuscript. This work relies on experimental results obtained at the Swedish National Infrastructure, DESIREE (Swedish Research Council Contract No. 2017-00621), originally published elsewhere, and I thank the DESIREE group at Stockholm University for collaboration on the experiments. This work is part of the project "Probing charge- and mass- transfer reactions on the atomic level," supported by the Knut and Alice Wallenberg Foundation (Grant No. 2018.0028), and supported by the Swedish Research Council through individual project grants with Contracts No. 2016-03765 and No. 2020-03404.

\section{APPENDIX: ONE-ELECTRON LCAO INTEGRALS FOR $l_{c}=l_{i}=0$}

The matrix element $T_{i c}(R)$ can be derived analytically using the asymptotic atomic wave functions, Eqs. (7) and (9), for the radial parts $\mathcal{R}_{c}$ and $\mathcal{R}_{i}$ of the asymptotic spatial atomic wave functions $\varphi_{c}$ and $\varphi_{i}$, respectively. The simplest possible case is taken, where both $\varphi_{c}$ and $\varphi_{i}$ are spherically symmetric and $l_{c}=l_{i}=0$. It is instructive to first consider the overlap, which can be shown to be

$$
S_{i c}(R)=\left\langle\varphi_{c} \mid \varphi_{i}\right\rangle=\frac{N_{i}}{\gamma_{i} R} \int_{0}^{R} d r r \mathcal{R}_{c}(r) e^{-\gamma_{i} R} \sinh \left(\gamma_{i} r\right)+\int_{R}^{\infty} d r r \mathcal{R}_{c}(r) e^{-\gamma_{i} r} \sinh \left(\gamma_{i} R\right),
$$

where $r=r_{A}$ (see Fig. 1). Performing these integrals with the aid of Mathematica, we obtain in its most compact form

$$
\begin{aligned}
S_{i c}(R)= & -\frac{N_{c} N_{i}}{2 \gamma_{i} R}\left[\left(\gamma_{c}-\gamma_{i}\right)\left(\gamma_{c}+\gamma_{i}\right)\right]^{-\frac{\gamma_{c}+1}{\gamma_{c}}} e^{-\gamma_{i} R}\left\{\left(\gamma_{c}-\gamma_{i}\right)^{\frac{1}{\gamma_{c}}+1}\left[e^{2 \gamma_{i} R} \Gamma\left(1+\frac{1}{\gamma_{c}},\left(\gamma_{c}+\gamma_{i}\right) R\right)-\Gamma\left(1+\frac{1}{\gamma_{c}}\right)\right]\right. \\
& \left.+\left(\gamma_{c}+\gamma_{i}\right)^{\frac{1}{\gamma_{c}}+1}\left[\Gamma\left(1+\frac{1}{\gamma_{c}}\right)-\Gamma\left(1+\frac{1}{\gamma_{c}},\left(\gamma_{c}-\gamma_{i}\right) R\right)\right]\right\},
\end{aligned}
$$

where $\Gamma(z)$ is the Euler gamma function and $\Gamma(a, z)$ is the incomplete gamma function (see, e.g., Ref. [34]). Similarly, $T_{i c}(R)$ can be evaluated simply by adding the operator $T=2\left(\frac{1}{r_{A}}-\frac{1}{R}\right)$ into Eq. (A1). Evaluating these integrals gives, in its most compact 
form,

$$
\begin{aligned}
T_{i c}(R)= & -\frac{N_{c} N_{i}}{\gamma_{c} \gamma_{i} R^{2}}\left[\left(\gamma_{c}-\gamma_{i}\right)\left(\gamma_{c}+\gamma_{i}\right)\right]^{-\frac{\gamma_{c}+1}{\gamma_{c}}} e^{-\gamma_{i} R}\left(2 \gamma_{c} \gamma_{i} e^{R\left(\gamma_{i}-\gamma_{c}\right)}\left[R\left(\gamma_{c}-\gamma_{i}\right)\left(\gamma_{c}+\gamma_{i}\right)\right]^{\frac{1}{\gamma_{c}}}\right. \\
& -\left\{\left(\gamma_{c}-\gamma_{i}\right)^{\frac{1}{\gamma_{c}}+1}\left[\gamma_{c} R\left(\gamma_{c}+\gamma_{i}\right)-1\right]\left[\Gamma\left(\frac{1}{\gamma_{c}}\right)-e^{2 \gamma_{i} R} \Gamma\left(\frac{1}{\gamma_{c}},\left(\gamma_{c}+\gamma_{i}\right) R\right)\right]\right\} \\
& \left.+\left(\gamma_{c}+\gamma_{i}\right)^{\frac{1}{\gamma_{c}}+1}\left[\gamma_{c} R\left(\gamma_{c}-\gamma_{i}\right)-1\right]\left[\Gamma\left(\frac{1}{\gamma_{c}}\right)-\Gamma\left(\frac{1}{\gamma_{c}},\left(\gamma_{c}-\gamma_{i}\right) R\right)\right]\right) .
\end{aligned}
$$

[1] T. Launoy, J. Loreau, A. Dochain, J. Liévin, N. Vaeck, and X. Urbain, Mutual neutralization in $\mathrm{Li}^{+}+\mathrm{D}^{-}$collisions: A combined experimental and theoretical study, Astrophys. J. 883, 85 (2019).

[2] G. Eklund, J. Grumer, S. Rosén, M. Ji, N. Punnakayathil, A. Källberg, A. Simonsson, R. D. Thomas, M. H. Stockett, P. Reinhed, P. Löfgren, M. Björkhage, M. Blom, P. S. Barklem, H. Cederquist, H. Zettergren, and H. T. Schmidt, Cryogenic merged-ion-beam experiments in DESIREE: Finalstate-resolved mutual neutralization of $\mathrm{Li}^{+}$and $\mathrm{D}^{-}$, Phys. Rev. A 102, 012823 (2020).

[3] G. Eklund, J. Grumer, P. S. Barklem, S. Rosén, M. Ji, A. Simonsson, R. D. Thomas, H. Cederquist, H. Zettergren, and H. T. Schmidt, Final-state-resolved mutual neutralization of $\mathrm{Na}^{+}$and $\mathrm{D}^{-}$, Phys. Rev. A 103, 032814 (2021).

[4] V. Vuitton, P. Lavvas, R. V. Yelle, M. Galand, A. Wellbrock, G. R. Lewis, A. J. Coates, and J. E. Wahlund, Negative ion chemistry in Titan's upper atmosphere, in Surfaces and Atmospheres of the Outer Planets, Their Satellites and Ring Systems: Part V, special issue of Planet. Space Sci., 57, 1558 (2009).

[5] E. Sagawa, T. J. Immel, H. U. Frey, and S. B. Mende, Longitudinal structure of the equatorial anomaly in the nighttime ionosphere observed by IMAGE/FUV, J. Geophys. Res. 110, A11302 (2005).

[6] P. S. Barklem, A. K. Belyaev, and M. Asplund, Inelastic H+Li and $\mathrm{H}^{-}+\mathrm{Li}^{+}$collisions and non-LTE Li I line formation in stellar atmospheres, Astron. Astrophys. 409, L1 (2003).

[7] P. S. Barklem, A. M. Amarsi, J. Grumer, G. Eklund, S. Rosén, M. Ji, H. Cederquist, H. Zettergren, and H. T. Schmidt, Mutual neutralization in $\mathrm{Li}^{+}+\mathrm{H}^{-} / \mathrm{D}^{-}$and $\mathrm{Na}^{+}+\mathrm{H}^{-} / \mathrm{D}^{-}$Collisions: Implications of experimental results for non-LTE modeling of stellar spectra, Astrophys. J. 908, 245 (2021).

[8] U. Fantz and D. Wünderlich, A novel diagnostic technique for $\mathrm{H}^{-}\left(\mathrm{D}^{-}\right)$densities in negative hydrogen ion sources, New J. Phys. 8, 301 (2006).

[9] C. Zener, Non-adiabatic crossing of energy levels, Proc. R. Soc. London, Ser. A 137, 696 (1932).

[10] D. R. Bates, Theoretical treatment of collisions between atomic systems, Pure Appl. Phys. 13, 549 (1962).

[11] T. C. Scott, A. Dalgarno, and J. D. Morgan, III, Exchange energy of $\mathrm{H}_{2}^{+}$Calculated from Polarization Perturbation Theory and the Holstein-Herring Method, Phys. Rev. Lett. 67, 1419 (1991).

[12] M. Chibisov and R. Janev, Asymptotic exchange interactions in ion-atom systems, Phys. Rep. 166, 1 (1988).
[13] D. M. Davidović and R. K. Janev, Resonant charge exchange of the negative ions in slow collisions with atoms, Phys. Rev. 186, 89 (1969).

[14] R. K. Janev and A. Salin, Exchange interaction between ionic and covalent states of two atoms at large distances, J. Phys. B 5, 177 (1972).

[15] R. K. Janev, On the long-range configuration interaction between ionic and covalent states, J. Chem. Phys. 64, 1891 (1976).

[16] O. B. Firsov, Resonant charge exchange between ions at slow collisions, Zh. Eksp. Teor. Fiz. 21, 1001 (1951).

[17] T. Holstein, Mobilities of positive ions in their parent gases, J. Phys. Chem. 56, 832 (1952).

[18] C. Herring, Critique of the Heitler-London method of calculating spin couplings at large distances, Rev. Mod. Phys. 34, 631 (1962).

[19] L. D. Landau and E. M. Lifshitz, Quantum Mechanics (Pergamon, Oxford, UK, 1965).

[20] B. M. Smirnov, Formation and decay of negative ions, Sov. Phys. Dokl. 10, 218 (1965).

[21] P. S. Barklem, Excitation and charge transfer in low-energy hydrogen-atom collisions with neutral atoms: Theory, comparisons, and application to Ca, Phys. Rev. A 93, 042705 (2016).

[22] P. S. Barklem, Erratum: Excitation and charge transfer in lowenergy hydrogen-atom collisions with neutral atoms: Theory, comparisons, and application to Ca [Phys. Rev. A 93, 042705 (2016)], Phys. Rev. A 95, 069906(E) (2017).

[23] R. K. Janev and Z. M. Radulovic, Ion-ion recombination and ion-pair formation processes in alkali-hydrogen diatomic systems, Phys. Rev. A 17, 889 (1978).

[24] R. K. Janev, C. L. Liu, J. G. Wang, and J. Yan, Mutual neutralization of $\mathrm{H}_{3}^{+}$and $\mathrm{H}^{-}$ions in slow collisions, Europhys. Lett. 74, 616 (2006).

[25] Wang Jian-Guo, Liu Chun-Lei, R. K. Janev, Yan Jun, and Shi Jian-Rong, Mutual recombination in slow $\mathrm{Si}^{+}+\mathrm{H}^{-}$collisions, Chin. Phys. 15, 2651 (2006).

[26] H. Miyano Hedberg, S. Nkambule, and Å. Larson, LandauZener studies of mutual neutralization in collisions of $\mathrm{H}^{+} / \mathrm{H}^{-}$ and $\mathrm{Be}^{+} / \mathrm{H}^{-}$, J. Phys. B 47, 225206 (2014).

[27] X. Zhou and A. S. Dickinson, Mutual neutralisation of $\mathrm{N}^{+}$by $\mathrm{O}^{-}$and $\mathrm{O}^{+}$by $\mathrm{O}^{-}$, Nucl. Instrum. Methods Phys. Res., Sect. B 124, 5 (1997).

[28] N. de Ruette, A. Dochain, T. Launoy, R. F. Nascimento, M. Kaminska, M. H. Stockett, N. Vaeck, H. T. Schmidt, H. Cederquist, and $\mathrm{X}$. Urbain, Mutual Neutralization of $\mathrm{O}^{-}$with 
$\mathrm{O}^{+}$and $\mathrm{N}^{+}$at Subthermal Collision Energies, Phys. Rev. Lett. 121, 083401 (2018).

[29] L. D. Landau, On the theory of transfer of energy at collisions I, Physika. Z. Sowietunion 1, 88 (1932).

[30] L. D. Landau, On the theory of transfer of energy at collisions II, Physika. Z. Sowietunion 2, 46 (1932).

[31] E. A. Andreev, Exchange interaction between two different atoms at large distances, Theor. Chim. Acta 30, 191 (1973).

[32] R. Grice and D. R. Herschbach, Long-range configuration interaction of ionic and covalent states, Mol. Phys. 27, 159 (1974).

[33] E. E. Nikitin and S. I. Umanskii, Theory of Slow Atomic Collisions, Springer Series in Chemical Physics, Vol. 30 (Springer, Berlin, 1984).

[34] NIST Handbook of Mathematical Functions, edited by F. W. J. Olver and NIST (Cambridge University Press, Cambridge, 2010).

[35] D. R. Hartree, The wave mechanics of an atom with a nonCoulomb central field. Part I. Theory and methods, Math. Proc. Cambridge Philos. Soc. 24, 89 (1928).

[36] D. R. Bates and A. Damgaard, The calculation of the absolute strengths of spectral lines, Philos. Trans. R. Soc. London, Ser. A 242, 101 (1949).

[37] M. J. Seaton, The quantum defect method, Mon. Not. R. Astron. Soc. 118, 504 (1958).

[38] B. M. Smirnov, Atomic structure and the resonant charge exchange process, Phys. Usp. 44, 221 (2001).

[39] R. K. Janev, Nonadiabatic transitions between ionic and covalent states, Adv. At. Mol. Phys. 12, 1 (1976).

[40] S. A. Adelman and D. R. Herschbach, Asymptotic approximation for ionic-covalent configuration mixing in hydrogen and alkali hydrides, Mol. Phys. 33, 793 (1977).

[41] S. D. Anstee, The collisional broadening of alkali spectral lines by atomic hydrogen, Ph.D. thesis, University of Queensland, 1992.

[42] B. M. Smirnov, Physics of Atoms and Ions, Graduate Texts in Contemporary Physics (Springer, New York, 2003).

[43] S. H. Patil, Asymptotic Methods in Quantum Mechanics: Application to Atoms, Molecules and Nuclei (Springer, Berlin, 2013).
[44] S. Patil, Asymptotic behaviour of wavefunctions: Its applications to properties of atoms, molecules and nuclei, Eur. J. Phys. 16, 25 (1995).

[45] K. R. Lykke, K. K. Murray, and W. C. Lineberger, Threshold photodetachment of $\mathrm{H}^{-}$, Phys. Rev. A 43, 6104 (1991).

[46] B. M. Smirnov, Asymptotic Methods in the Theory of Atomic Collisions [in Russian] (Nauka, Moscow, 1973).

[47] S. Chandrasekhar, Some remarks on the negative hydrogen ion and its absorption coefficient, Astrophys. J. 100, 176 (1944).

[48] C. L. Pekeris, Ground state of two-electron atoms, Phys. Rev. 112, 1649 (1958).

[49] T. Ohmura and H. Ohmura, Electron-hydrogen scattering at low energies, Phys. Rev. 118, 154 (1960).

[50] T. Ohmura, Y. Hara, and T. Yamanouchi, Low energy electronhydrogen scattering, Prog. Theor. Phys. 20, 82 (1958).

[51] J. F. Hart and G. Herzberg, Twenty-parameter eigenfunctions and energy values of the ground states of $\mathrm{He}$ and He-like ions, Phys. Rev. 106, 79 (1957).

[52] E. A. Hylleraas, Neue Berechnung der Energie des Heliums im Grundzustande, sowie des tiefsten Terms von Ortho-Helium, Z. Phys. 54, 347 (1929).

[53] X.-Y. Pan, V. Sahni, and L. Massa, On the Hylleraas coordinates, arXiv:physics/0310128.

[54] H. Croft, A. S. Dickinson, and F. X. Gadéa, A theoretical study of mutual neutralization in $\mathrm{Li}^{+}+\mathrm{H}^{-}$collisions, J. Phys. B 32, 81 (1999).

[55] H. Croft, A. S. Dickinson, and F. X. Gadéa, Rate coefficients for the $\mathrm{Li}^{+} / \mathrm{H}^{-}$and $\mathrm{Li}^{-} / \mathrm{H}^{+}$mutual neutralization reactions, Mon. Not. R. Astron. Soc. 304, 327 (1999).

[56] A. S. Dickinson, R. Poteau, and F. X. Gadéa, An ab initio study of mutual neutralization in $\mathrm{Na}^{+}+\mathrm{H}^{-}$collisions, J. Phys. B 32, 5451 (1999).

[57] A. Dochain and X. Urbain (private communication).

[58] The exchange interaction is used in two senses here, in $\mathrm{H}_{2}$ as the usual interaction due to antisymmetrization with respect to exchange of electron labels and in $\mathrm{H}_{2}^{+}$due to the degeneracy with respect to the exchange of position of the electron from one nucleus to the other. 